\title{
Ixerides Japonicae
}

auctore

\section{Siro Kitamura}

Received October 12, 1934

Ixeris Cass., Bull. Philom. (1821) p. 173, Dict. des Sci. Natur. XXIV (1822) p. 49, DC., Prodr. VII (1838) p. 151, NakaI, in Bot. Mag. Tokyo XXXVI (1922) p. 24.

Syn. Chorisma Don, in Edin. N. Phil. Journ. 1828-29 p. 308 non Lindl. (1820).

Chorisis DC., Prodr. VII (1838) p. 177.

Lactuca sect. Ixeris Benth. et Hooker pro parte et sect. Chorisma Benth. et Hooker, Gen. Pl. II (1873) p. 526, Franchet, in Journ. de Bot. IX (1885) p. 261, Hofrmann, in Pflanzenfamilien IV-5 (1894) p. 372.

Capitula liguliflora. Involucrum cylindricum, squamae pauciseriales imbricatae membranaceo-herbaceae pruinosae glabrae, post anthesin immutatae, intimae quam extimae multo-longiores, exteriores saepe calyculatae minutae vel gradatim longiores. Receptaculum planum nudum. Antherae basi sagittatae. Achenia vix compressa elongato-oblonga acute 10-alata saepe longe in rostrum sensim abientia. Pappi setae candidae vel sordidae copiosae tenuissimae minute scabrae subaequilongae. Herbae asiaticae annuae, biennes vel perennes humiles, folia radicalia sub anthesin rosulata, capitula corymbosa vel oligocephala, corolla flava, alba vel violacea.

Sect. Chorisis (DC.) Asa Gray, on the Botany of Japan (1859) p. 379.

Syn. Chorisma Don, in Edin. N. Phil. Journ. 1828-29 p. 304 non Lindl. (1820).

Chorisis DC. Prodr. VII (1838) p. 177.

Lactuca sect. Chorisma Benth. et Hook., Gen. Pl. II (1873) p. 526, Hoffman, in Pflanzenfamilien IV-5 (1894) p. 372.

Capitula multiflora, involucrum latiusculum cire. $11 \mathrm{~mm}$. longum $8 \mathrm{~mm}$. latum in sicco, squamae exteriores inaequilongae sub-biseriales majores, intimae saepe ad medium interiorum attingentes. Achenia 5.5-6 mm. longa breve rostrata, rostrum vix $1 \mathrm{~mm}$. longum. Caulis longe repens, capitula laxe corymbosa oligocephala.

Ixeris repens (L.) Asa Gray, 1. c. p. 397, Benth. Fl. Hongk. (1861) 
p. 194, Fr. et Sav., Enum. Pl. Jap. I(1875) p. 271, Nakai, Fl. Sylv. Koreana XIV (1923) p. 114.

Syn. Prenanthes repens L. Sp. Pl. (1753) p. 798.

Chorisis repens DC. Prodr. VII (1838) p. 178.

Nabalus repens Ledeb., Fl. Ross. II (1846) p. 840.

Lactuca repens Bentir., Maxim. Mél. Biol. IX (1874) p. 364, Matsum., Index Pl. Jap. II (1912) p. 654, IIAyata, Comp. Formos. (1904) p. 40, Hultén, Fl. Kamtsch. IV (1930) p. 231.

Lactuca brachyrhyncha Hayat', Ic. Pl. Formos. VIII (1919) p. 74. Syn. Nov.

Nom. jap. Hama-nigana.

Hab. Kamtschatca, Kuriles, Sachalin, Hokkaido, Hondo, Shikoku, Kiushiu, Liukiu, Formosa, Manshuria, China.-in litoribus maritimis crescens.

Sect. Ixeridium Asa Gray, l. c. p. 379.

Capitula pauciflora. Involucrum cylindricum angustum $5-9 \mathrm{~mm}$. longum, 1-4 mm. latum in sicco, squamae exteriores uniseriatim dispositae minimae calyculatae subaequales, interiorum $\frac{1}{5}-\frac{1}{i}$ atingentes. Achenia angusta $3-6 \mathrm{~mm}$. longa, $\frac{1}{2} \mathrm{~mm}$. lata, rostrum $\frac{1}{2}-3 \mathrm{~mm}$. longum, alae obtusiusculae. Caulis saepissime erectus rarius repens, capitula polycephala laxe corymbosa.

$\{$ Achenia longe rostrata, rostrum achenio aequilongum ......... II.

I. $\left\{\right.$ Achenia breve rostrata, rostrum $\frac{1}{3}-\frac{1}{5}$ achenii aequilongum ........ IV. , Capitula angusta $3 \mathrm{~mm}$. It. in sicco, folia crassiuscula pappus sordidus II. $\{\ldots \ldots \ldots \ldots \ldots \ldots \ldots \ldots \ldots .1$. Ixeris Oldhami (Maxim.) Kitamura. Capitula latiora $4 \mathrm{~mm}$. lt. in sicco, folia tenuia, pappus candidus .... Folia radicalia copiosa lineari-lanceolata plerumque integra .......

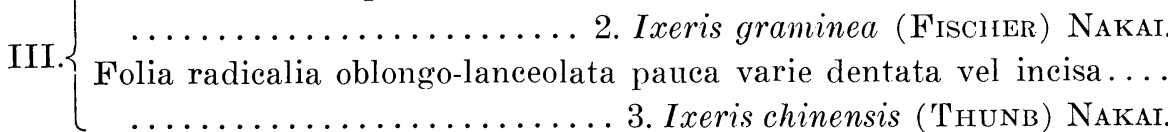
(Involucrum sub anthesin $4 \mathrm{~mm}$. longum, achenia $3 \frac{1}{2} \mathrm{~mm}$. longa, folia linearia ............. Ixeris Makinoana (Kiтам.) KiтAм.

IV. $\{$ Involucrum sub anthesin 7-9 $\mathrm{mm}$. longum, achenia $5 \mathrm{~mm}$. longa, folia

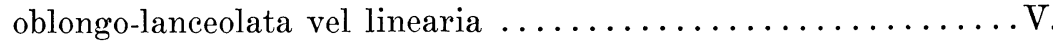

V. $\left\{\begin{array}{l}\text { Folia linearia crassiuscula 5. Ixeris transnokoensis (SASAKi) Kitamura. } \\ \text { Folia oblongo-lanceolata tenuia....6. Ixeris dentata (Thunb.) NaKai. }\end{array}\right.$

1. Ixeris Oldhami (Maxim.) Kitanura, in Acta Pytotax. et Geobot. III (1934) p. 134 
Syn. Lactuca Oldhami Maxim., Mél. Biol. IX (1874) p. 363, Hayata, Ic. Pl. Formos. VIII (1919) p. 76, Takeda, in Bot. Mag. Tokyo XXIV (1910) p. (348).

Lactuca dentata Makino var. lanceolata Makino, in Bot. Mag. Tokyo XXVIII (1913) p. 30.

Lactuca stenophylla Makino, in Journ. Jap. Bot. IV (1927) p. 10.

Lactuca dentata Robinson, in Philip. Journ. Sci. III (1908) p. 218 excl. syn., Merril, Enum. Philip. Pl. III (1923) p. 621.

Lactuca Thunbergiana Forbes et Hemss. (non Maxim.), in Journ. Linn. Soc. XXIII (1888) p. 484 excl. syn. pro parte, Elmer, Leafl. Philip. Bot. I (1906) p. 183.

Nom. jap. Atsuba-nigana, Yanagi-nigana.

Hab. Kiushiu australis, Liukiu, Formosa, Philippine.-in ag'ris argillaceis occurrit.

Ixeris Gregorii HaNCE in Ann. Soc. Nat. 5me serie V. (1866) p. 222 mihi ignota.

2. Ixeris graminea (FIscher) NAKaI in Bot. Mag. Tokyo XXXVI (1922) p. 23, Fl. Sylv. Koreana XIV (1923) p. 114.

Syn. Prenanthes graminea Fischer in Mém. Soc. Nat. Mosc. III (1812) p. 67.

Lactuca Fischeriana DC., Prodr. VII (1838) p. 135.

Lactuca versicolor var. arenicola MAkino, in Bot. Mag. Tokyo XII (1898) p. 44.

Lactuca tamagawaensis Makino, l. c. VI (1892) jap. p. 56, XVIII (1903) p. 90.

Lactuca gracilis Hayata (non DC.), Comp. Formos. (1904) p. 40.

Lactuca taitoensis Hayata, Ic. Pl. Formos. VIII (1919) p. 76, fig. 31-2. Syn. Nov.

Nom. jap. Kawara-nigana, Taito-takasagoso.

Hab. Siberia, Manshuria, Korea, Honshu, Formosa.-in arenas agrorum vel riporum crescit.

3. Ixeris chinensis (Thunb.) NakaI, in Bot. Mag. Tokyo XXXIV (1920) p. 152. Kitagawa, l. c. XLVIII (1934) p. 113 excl. syn. et specim.

Syn. Prenanthes chinensis Thunb., Fl. jap. (1784) p. 301 pro parte.

Chondrilla chinensis PoIret, Encycl. Méth. Suppl. II (1811) p. 331.

Youngia chinensis DC., Prodr. VII (1838) p. 194.

Ixeris versicolor Benth. (non DC.) Fl. Hongk. (1861) p. 193.

Lactuca versicolor Maxim. (non Schltz Bir.), Mél. Biol. IX (1874) p. 362 excl. syn. et specim. pro parte, Forbes et HemsL., in Journ. Linn. 
Soc. XXIII (1888) p. 485, Hayata, Comp. Formos. (1904) p. 39, Gagnep., in Lecomte, Fl. général Indo-Chine III (1924) p. 656.

Lactuca flavissima Hayata, Ic. Pl. Formos. VIII (1919) p. 78 fig. 31-3. Lactuca chinensis Yамамото (non Makino), in Journ. Trop. Agric. III (1931) p. 243.

Lactuca lacerrima Hayata, forma flavissima Kitamura in Acta Phytotax. Geobot. I (1932) p. 152.

Nom. jap. Usagi-so.

Hab. Liukiu, Formosa, China australis, Indo-China.-in agris argillaceis frequens.

var. saxatilis (Kitamura) Kitamura comb. nov.

Syn. Lactuca lacerrima Hayata var. saxatilis Kitamura, l.c. p. 152.

Lactuca lacerrima Hayata, Ic. Pl. Formos. VIII (1919) p. 76, KitaMURA, l. c. p. 151.

Radix ramosa, caulis humilis, folia margine ciliato-serrulata, flores flavae.

Nom. jap. Kireba-takasagoso.

Hab. Formosa : Prov. Karenko.-in fissis saxorum erescens.

subsp. versicolor (SPREngel) Kitamura comb. nov.

Barkhausia versicolor Sprengeu, Syst. Veg. III (1826) p. 651.

Prenanthes versicolor Fiscier ex Bunge in Mém. Sav. Etrang. Acad. Sci. St.-Pétersb. II (1833) p. 114.

Ixeris versicolor DC., Prodr. VII (1838) p. 151, LedeB., Fl. Ross. II (1844-46) p. 817, Maxim., Prim. Fl. Amur. (1859) p. 180, 473, 483.

Lactuca versicolor Schultz BiP. ex Herder, Pl. Radd. III. 4 (1870) p. 29, Maxim., Mél. Biol. IX (1874) p. 362 pro parte, Forbes et Hemsl., in Journ. Linn. Soc. XXIII (1888) p. 485 pro parte, Komarov, Fl. Mansh. III (1907) p. 782 pro parte, NAKAI, Fl. Koreana II (1911) p. 56.

Lactuca hallaisanensis Lévl. in Fedde Rep. XII(1913) p. 100. Syn. Nov. Ixeris chinensis NakaI (non NakaI in 1922), Fl. Sylv. Koreana XIV (1923) p. 113, Kitagawa, in Bot. Mag. Tokyo XLVIII (1934) p. 113 pro parte.

Nom. jap. Manshu-takasagoso n. n.

Hab. Korea, Manshuria, Siberia, China-borealis.-in agris argillaceis frequens.

subsp. strigosa (Lével. et Vaniot.) Kitamura comb. nov.

Syn. Lactuca strigosa LÉvel. et VANiot. in Bull. Acad. Géogr. Bot. XX Mém. (1909) p. 114.

Prenanthes chinensis Thunb. 1. c. p. 301 pro parte.

Lactuca chinensis Makino, in Bot. Mag. Tokyo XVII (1903) p. 89, 
Matsum., Index Pl. Jap. II (1912) p. 652.

Lactuca albiflora NAKaI (non Asa GraY), in Bot. Mag. Tokyo XXIV (1910) jap. p. 267.

Lactuca chinensis Tanaka in Bult. Sci. Fakult. Terkult. Kjusu Imp. Univ. I (1925) p. 205.

Ixeris versicolor Miq. (non DC.), Ann. Musei Bot. Lugd.-Bat. II(1866) p. 191, Franchet et Sav., Enum. Pl. Jap. I (1875) p. 269.

Nom. jap. Takasagoso.

Hab. Hondo, Shikoku, Kiushiu, Korea.-in declinitate graminoso occurrit.

Clavis subspecierum.

1. Caulis elatus circ. $25-35 \mathrm{~cm}$. altus, radix saepe ramosa, involucrum 6-8 mm. longum, corolla semper flavissima ....... Ixeris chinensis NAKAI.

2. Caulis humilis cire $15-25 \mathrm{~cm}$. altus, radix saepe ramosa, involucrum 8-9 mm. longum, corolla flava, violacea vel albescens..Ixeris versicolor DC.

3. Caulis elatus circ. $30-40 \mathrm{~cm}$. altus, radix plerumque simplex, involucrum 8-10 mm. longum, corolla semper albo-violacea

4. Ixeris Makinoana (Kitamura) Kitamura comb. nov.

Syn. Lactuca Thunbergii Maxim., var. angustifoila Makino, in Bot. Mag. Tokyo XIX (1905) p. 154.

Lactuca dentata Makino a. flaviflora Makino subvar. angustifolia Makino l. c. XXIV (1910) p. 75, Matsum., Index Pl. Jap. II (1912) p. 653.

Lactuca Makinoana Kitamura, Comp. Nov. Jap. I (1931) p. 26.

Nom. jap. Hosoba-nigana.

Hab. Kiushiu, Shikoku, Hondo australis.

5. Ixeris transnokoensis ( SASAKI) KitamURA comb. nov.

Syn. Lactuca transnokoensis SASAKI in Trans. Nat. Hist. Soc. Formos. Vol. XXI (1931) p. 223.

Rhizoma elongatum circ. $1 \mathrm{~mm}$. in diametro, radicibus filiformibus paucis emittens. Caulis $8-20 \mathrm{~cm}$. altus gracilis ascendens non repens, glaber ramosus. Folia radicalia rosulata sub anthesin viva, linearia $4-8.5 \mathrm{~cm}$. longa circ. $4 \mathrm{~mm}$. lata apice acuta basi longe in petiolum attenuata utrinque uninerviata glabra crassiuscula, margine plerumque integra, rarius ciliatoserrata, folia caulina superiora pauca anguste linearia parva. Capitula laxe corymbosa gracile pedunculata, involucrum nigro-viridescens anguste cylindricum $7.5 \mathrm{~mm}$. longum basi minutissime calyculatum. Squamae interiores 5, lineares albo-marginatae. Achenia $4 \mathrm{~mm}$. longa apice rostro $1 \mathrm{~mm}$. longo attenuata, 10-costata. Pappus sordidus $5 \mathrm{~mm}$. longus. Flos- 
culus flavus $10 \mathrm{~mm}$. longus $3 \mathrm{~mm}$. latus, pars tubi $2 \mathrm{~mm}$. longa.

Nom. jap. Miyama-nigana.

Hab. Formosa in zona alpina frequens, Prov. Karenko: inter mt. Kiraisu et Noko (24 aug. 1929 S. SASAKI), Prov. Tainan : mt. Niitaka inter Tataka et Niitakashita (5 jul. 1933 J. OHwi), Prov. Takao: Kanzangoe (28 aug. 1933 K. Koлгмa), Prov. Taichu: Hattsukan et Rakuraku (9 jul. 1933 J. OHwi).

6. Ixeris dentata (Thunb.) NakAI, Fl. Sylv. Koreana XIV (1923) p. 114.

Syn. Prenanthes dentata Thunb., Fl. jap. (1784) p. 301.

Chondrilla dentata Poiret, Encycl. Méth. suppl. II (1811) p. 328.

Youngia dentata DC., Prodr. VII (1838) p. 193 excl. syn.

Ixeris Thunbergii Asa GraY, on the Botany of Japan (1859) p. 397, Miq., in Ann. Musei Bot. Ludg.-Bat. II. (1866) p. 191, Fr. et SAv., Enum. Pl. Jap. I (1875) p. 270.

Lactuca Thunbergii Maxim., Mél. Biol. IX (1874) p. 361.

Lactuca dentata RoBinson, in Philip. Journ. Sci. III (1908) p. 218 excl. specim.

Lactuca dentata Makino, in Bot. Mag. Tokyo XXIV (1910) p. 75, Matsun., l. c. p. 653, Takeda, in Bot. Mag. Tokyo, XXIV (1910) p. (348).

Nom. jap. Nigana.

Hab. Hokkaido, Kuriles, Hondo, Shikoku, Kiushiu, Korea, Liukiu.in siccis graminosis vel in agris frequens.

var. albiflora (MAKINO) NAKAI, Fl. Svlv. Koreana XIV (1923) p. 114.

Syn. Lactuca Thunbergii Maxim., var. albiflora Makino in Bot. Mag. Tokyo XII (1898) p. 48.

Lactuca dentata Makino var. albiflora Makino, in Bot. Mag. Tokyo XXIV (1910) p. 76, Matsum., l. c. p. 653.

Nom. jap. Shirobana-nigana.

Hab. ut in typico.

var. atro-purpurea NAKAI, in Bot. Mag. Tokyo XLII (1928) p. 16.

Nom. jap. Kuro-nigana.

Hab. in holto culta.

var. amplifolia Kitamura var. nov.

Caulis robustior, folia latiora, radicalia ambitu late oblonga vel elliptica apice obtusa mucronulata basi in petiolum elongatum alato-decurrentia, caulina media oblonga basi ciliato-serrata late amplexicaulia, superiora ovata basi dilatata. Floseuli 10-11.

Nom. jap. O-nigana n. n. 
Hab. Hondo: Prov. Yamashiro: Uji (23 maius 1933 S. Kitamura). —Typus!, Hondo, Shikoku.-cum typico mixte erescens.

var. partita (Makino) Kitamura comb. nov.

Syn. Lactuca dentata var. Thunbergii f. partita Makino, in Bot. Mag. Tokyo XXVII (1913) p. 29.

Lactuca dentata var. partita Makino, 1. c. XXVIII (1914) p. 174.

Nom. jap. Kikuba-nigana.

Hab. Kiushiu, Hondo.

subsp. alpicola (Nakai) Kitamura comb. nov.

Syn. Lactuca Thunbergii Maxim. lusus alpicola TAKedA, in Bot. Mag. Tokyo XXIV (1910) p. 70.

Lactuca dentata $\alpha$. flaviflora subvar. alpicola Makino, l. c. p. 75.

Lactuca alpicola NAKAI, l. c. jap. p. 267.

Lactuca dentata var. alpicola Makino, 1. c. XXVII (1913) p. 29.

Ixeris alpicola NAKaI, l. e. XXXVI (1922) p. 23.

Nom. jap. Takane-nigana.

Hab. Hondo, Shikoku, insl. Yaku.-in zona alpina crescit.-Praecertim in Mt. Yatsugatake, Mt. Howoo, Mt. Fuji et Mt. Shirane, acheniorum rosta longiora gracilia.

subsp. Kimurana Kitamura in Acta Phytotax. Geobot. III (1934) p. 133.

Nom. jap. Kumoma-nigana.

Hab. Hondo borealis in zona alpina occurrit.

var. leucantha Kitamura nom. nov.=var. albiflora Kitaм. 1. c. p. 134.

Nom. jap. Shirobana-kumoma-nigana.

Hab. cum typico occurrit.

subsp. nikoensis (NAKAI) Kitamura comb. nov.

Syn. Ixeris albiflora Asa Gray, on the Bot. Jap. (1859) p. 397.

Lactuca albiflora Maxim., Mél. Biol. IX (1874) p. 361.

Ixeris nikoensis NakaI, in Bot. Mag. Tokyo XLIV (1930) p. 23.

Lactuca dentata var. stolonifera Kitamura, Comp. Nov. Jap. (1931) p. 26.

Nom. jap. Hai-nigana, Tsuru-kawara-nigana.

Hab. Hondo, Kiushiu ubique rara.-in agris vel ripis crescit.Corolla flava, in sicco albescens.

Clavis subspecierum.

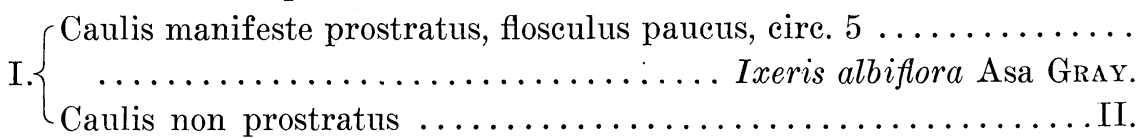


Herba sylvestris vel oleracea, caulis elatus, capitula parva, folia radi-

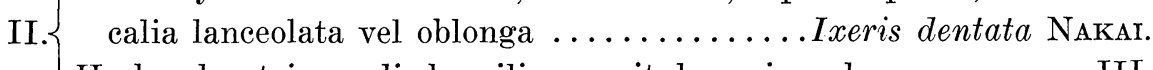
Herba alpestris, caulis humilior, capitula majuscula .......... III.

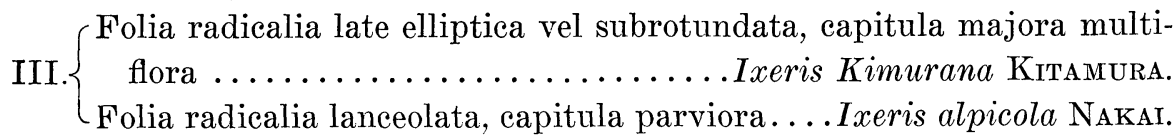

Sect. Pseudo-chorisis Kitamura sect. nov.

Capitula multiflora, involucrum cylindricum latiusculum 8-14 mm. longum $6-8 \mathrm{~mm}$. latum in siceo, squamae exteriores laxe calyculatae interiorum $\frac{1}{3}-\frac{1}{4}$ attingentes. Achenia latiuscula $5-9 \mathrm{~mm}$. $1 \mathrm{~g}$. longe rostrata profunde sulcata, alis acutis. Caulis semper stoloniferus, capitula oligocephala laxe corymbosa flava.

Involucrum circ. $8 \mathrm{~mm}$. longum, folia parva, achenia longissime rostrata ..1. Ixeris stolonifera Asa Gray.

Involucrum circ. $14 \mathrm{~mm}$. longum, folia majora, achenia longe rostrata 2. Ixeris japonica (BurmanN) NaKaI.

1. Ixeris stolonifera Asa GrAy, on the Bot. of Jap. (1859) p. 396, Miq., in Ann. Musei Bot. Lugd.-Bat. II (1866) p. 191, Fr. et SAv:, Enum. Pl. Jap. I (1875) p. 271, NakaI, Fl. Sylv. Koreana XIV (1923) p. 114.

Syn. Lactuca stolonifera Bentri., Maxim., Mél. Biol. IX (1874) p. 363, Forbes \& Hemsl., in Journ. Linn. Soc. XXIII (1888) p. 484, Matsumura, Index Pl. Jap. II (1912) p. 655.

Nom. jap. Iwa-nigana, Hai-jishibari.

Hab. Hokkaido, Hondo, Shikoku, Kiushiu, Korea, China.--in agris argillaceis crescens.

subsp. capillaris (NAKAI) KITAMURA comb. nov.

Syn. Lactuca stolonifera var. sinuata Makino, in Journ. Jap. Bot. III (1926) p. 42 ?

Ixeris capillaris NAKaI, Kamikochi-Tennenkinenbutsu-Chosahokoku (1928) p. 41.

Herba alpestris gracilis longe stolonifera, folia parva oblonga petiolata, ambitu 10-20 mm. longa 7-10 mm. lata, saepe sinuato-dentata vel lobata interdum pinnati-partita, capitula oligocephala, involucrum circ. $8 \mathrm{~mm}$. longum, achenia ut in typico.

Nom. jap. Miyama-iwanigana, Kikuba-jishibari.

Hab. Hondo borealis in zona alpina occurrit.-Mt. Tateyama, Mt. Hakusan, Mt. Iwate.

Clavis subspecierum.

1. Folia ampla ambitu saepe rotundata subintegra $\ldots \ldots \ldots \ldots \ldots$ 
2. Folia minora ambitu saepe oblonga sinuato-lobata $\ldots \ldots \ldots \ldots$ ....................... Ixeris capillaris NAKAI.

2. Ixeris japonica (BurmanN) NakaI, in Bot. Mag. Tokyo XL (1926) p. 574, Kitagawa, l. c. XLVIII (1934) p. 112.

Syn. Lampsana japonica Burmann, Fl. Ind. (1768) p. 174, tab. 57-1 excl. syn.

Prenanthes debilis Thunb., Fl. jap. (1784) p. 300, Ic. Pl. Jap. Decas IV (1802) t. 9.

Chondrilla debilis PoIRet, Encycl. Méth. Bot. suppl. II (1811) p. 332.

Youngia debilis DC., Prodr. VII (1838) p. 194.

Ixeris debilis A. Gray, l. c. p. 397, Benth. Fl. Hongk. (1861) p. 193 excl. specim., Miq., in Ann. Musei Bot. Lugd.-Bat. II (1866) p. 191, Fr. et Sav., Enum. Pl. Jap. I (1875) p. 270, NaKaI, Fl. Sylv. Koreana XIV (1923) p. 114.

Lactuca debilis Benth., Maxim., Mél. Biol. IX (1874) p. 365, Matsum., 1. c. p. 653 .

Nom. jap. Jishibari, Tsurunigana.

Hab. Hokkaido, Hondo, Shikoku, Kiushiu, Korea.-in agris argillaceis crescit.

f. dissecta NakaI, in Bot. Mag. Tokyo XL (1926) p. 576.

Hab. insula Quelpaert.

f. integra NAKAI, l. c. p. 575 .

Hab. Korea, Kiushiu, Liukiu.

f. sinuata NAKAI, l. c. p. 576.

Syn. Ixeris debilis $\beta$. sinuata Fr. et SAv., l. c. p. 270.

Hab. Hondo, Shikoku, Kiushiu, Korea, Liukiu, Bonin.

subsp. litoralis Kitamura, in Acta Phytotax. Geobot. III(1934) p. 132.

Syn. Ixeris debilis Benth. (non Asa GrAY), Fl. Hongk. (1861) p. 193 pro parte.

Lactuca debilis Hayata (non Benth.), Comp. Formos. (1904) p. 40, Ic. Pl. Formos. VIII (1919) p. 76.

Ixeris japonica NakaI var. salsuginosa Kittagawa, in Bot. Mag. Tokyo XLVIII (1934) p. 113.

Nom. jap. Hosoba-jishibari, Manshu-jishibari.

Hab. Formosa, China, Manshuria.-in litoribus martimis erescit.

subsp. trifida (Kitamura) Kitamura comb. nov.

Syn. Lactuca trifida Kitamura, in Act. Phytotax. Geobot. I (1932) p. 58.

Nom. jap. Mitsuzake-jishibari.

Hab. Formosa.-in litoribus maritimis occurrit. 
var. Nakazonei Kitamura var. nov.

Folia magna $12-20 \mathrm{~cm}$. longa, $4-6 \mathrm{~cm}$. lata, oblonga longe petiolata vel obovata breve petiolata, varie dissecta, textura crassiuscula.

Nom. jap. Miyako-jishibari n. n.

Hab. Liukiu : insl. Miyako (leg. Y. Nakazone 1933)—Typus!, insula Utchina prope Naha (14 Maius 1923 G. KoIdzumI).

Clavis subspecierum.

1. Folia spatulato-lanceolata apice rotundata..Ixeris japonica NAKAI.

2. Folia lineari-lanceolata interdum pinnatifida apice acuta textura erassiuscula ................. Ixeris litoralis Kitamura.

3. Folia oblonga trifida vel varie dissecta.... Ixeris trifida Kitamura.

Sect. Indo-ixeris Kitamura sect. nov.

Involucrum late cylindricum sub anthesin $5 \mathrm{~mm}$. longum, $3 \mathrm{~mm}$. latum, basi squamellis minutissimis 3-4 indistincte calyculatum. Achenia elliptica profunde sulcata, alis acutis praedita, longe rostrata. Capitula polycephala pseudo-umbellatim disposita, in fructu cernua, Caulis ramosus erectus, folia lanceolata, caulina basi sagittata apice acuminata.

Ixeris polycephala CAss., in Dict. Se. Nat. XXIV (1822) p. 50, DC., Prodr. VII (1838) p. 151, NakaI, in Bot. Mag. Tokyo XXXVIII (1924) jap. p. 265.

Syn. Lactuca polycephala Benth, Benth. et Hook., Gen. Pl. II (1873) p. 526, Clarke, Comp. Ind. (1876) p. 272, Hooker, Fl. Brit. Ind. III (1881) p. 410, Forbes \& Hemsu., in Journ. Linn. Soc. XXIII (1888) p. 483, Franth., Pl. David. (1884) p. 188.

Lactuca Matsumurae Makino, in Bot. Mag. Tokyo VI (1892) p. 56nomen nudum, XII (1898) p. 45, Matsum., Index Pl. Jap. II (1912) p. 654, Kitamura, in Acta Phytotax. Geobot. I (1932) p. 152.

Lactuca biauriculata LÉvel. et VANiot, in Bull. Acad. Géogr. Bot. XX Mém. (1909) p. 143.

Ixeris Matsumurae NaKaI, in Bot. Mag. Tokyo XXXIV (1920) p. 153, Fl. Sylv. Koreana XIV (1923) p. 114.

Nom. jap. Nonigana.

Hab. Hondo, Shikoku, Kiushiu, Korea, Liukiu, Formosa, China australis, Burma, Bengalia, Assamia, Sikkim, Nepalia, Affghanistan, Caucasia.--in agris argillaceis crescit.

var. dissecta (Makino) NaKaI, l. c. jap. p. 265.

Syn. Lactuca Matsumurae Makino var. dissecta Makino, in Bot. Mag. Tokyo XXIV (1910) p. 252.

Nom. jap. Kikuba-nonigana.

Hab. Korea, Kiushiu, Nepalia. 